\title{
Outpatient kidney recovery after acute kidney injury requiring dialysis: a systematic review protocol
}

\author{
Carol Wang ${ }^{1}$, Swapnil Hiremath ${ }^{2}$, Lindsey Sikora ${ }^{3}$, Manish M. Sood ${ }^{2}$, Jennifer Kong ${ }^{4}$ and Edward Clark ${ }^{2^{*}}$ (D)
}

\begin{abstract}
Background: Acute kidney injury requiring dialysis (AKI-D) during hospitalization is associated with both in-hospital and post-discharge mortality. Its incidence has risen over time in Canada and the USA. While the majority of AKI-D will recover to dialysis independence at the time of hospital discharge, 10-30\% will transition to outpatient dialysis. The risk factors that determine dialysis independence after AKI-D and its optimal outpatient management remain unclear. Eliciting prognostic predictors of kidney recovery in patients who remain on dialysis after hospital discharge will guide subsequent clinical decision making. The objective of this study is to assess the association between patient- and treatment-related factors with short- and long-term outcomes in patients who remained dialysisdependent after hospitalization with AKI-D.

Methods: A literature search in EMBASE, MEDLINE, and PubMed will be performed based on pre-specified criteria. There are no restrictions on language and publication dates. The supplemental search will include manual scan of bibliographies of eligible studies and grey literature assessment. Pre-specified criteria will be used to select eligible studies. Relevant data will be extracted and quality assessments performed per validated tools. Qualitative data synthesis will be performed to reflect directions of associations. Meta-analysis will be formed if two or more studies with similar prognostic factors, outcomes, and adequate quality are identified. Strength of association will be quantified as odds ratios. Reporting of this review will be guided by recommendations of the Preferred Reporting Items for Systematic Reviews and Meta-Analyses (PRISMA) statement.
\end{abstract}

Discussion: This systematic review aims to synthesize association between modifiable and non-modifiable prognostic factors with renal outcomes in AKI-D patients who remain dialysis-dependent after hospital discharge. Our findings will help inform the development of evidence-based management and guide long-term treatment planning for AKI-D patients.

Systematic review registration: PROSPERO registration number CRD42019127394.

Keywords: Acute kidney injury, Dialysis, Prognostic factors, Kidney recovery, Systematic review

\section{Background}

Acute kidney injury requiring dialysis (AKI-D) occurs in $1-2 \%$ of hospitalized patients $[1,2]$. In the USA, the incidence of AKI-D has increased in the span of 2000-2014, especially in patients with diabetes [3]. A retrospective population-based study in Ontario, Canada, demonstrated a fourfold increase in AKI-D from 0.8 to 3\% between 1996

\footnotetext{
* Correspondence: edclark@toh.ca

${ }^{2}$ Division of Nephrology, Department of Medicine, The Ottawa Hospital and University of Ottawa, The Ottawa Hospital - Riverside Campus, 1967

Riverside Drive, Ottawa, Ontario K1H 7 W9, Canada

Full list of author information is available at the end of the article
}

and 2010 [4]. AKI-D is also associated with increased risk of both in-hospital and post-discharge mortality $[1,5]$. Approximately $10-30 \%$ of patients with AKI-D who survive to hospital discharge will require outpatient dialysis [6]. Furthermore, 1 in 12 of those patients with kidney recovery to dialysis independence at the time of discharge will eventually need to resume it within 3 to 5 years' time [1]. The factors that determine the extent of kidney recovery after AKI-D remain unclear and are likely to consist of a range of modifiable and non-modifiable factors [2].

(C) The Author(s). 2019 Open Access This article is distributed under the terms of the Creative Commons Attribution 4.0 International License (http://creativecommons.org/licenses/by/4.0/), which permits unrestricted use, distribution, and reproduction in any medium, provided you give appropriate credit to the original author(s) and the source, provide a link to the Creative Commons license, and indicate if changes were made. The Creative Commons Public Domain Dedication waiver (http://creativecommons.org/publicdomain/zero/1.0/) applies to the data made available in this article, unless otherwise stated. 
An improved understanding of predictors of kidney recovery to dialysis independence in outpatients who require ongoing dialysis after a hospital encounter with AKI-D (as well as the gaps in knowledge in this area) can improve patient-centered decision-making and clarify the need for further research in this area. For patients unlikely to have kidney recovery, earlier and better-informed decision-making regarding long-term arteriovenous access, home therapies, and transplantation would be facilitated. This could improve patients' quality of care; improve access, integration, and utilization of chronic kidney disease system resources; and improve access to transplant and home dialysis. For caregivers of patients more likely to recover, frequent assessment for recovery and strategies to limit further kidney damage (e.g., avoidance of intradialytic hypotension and nephrotoxins) may facilitate shorter recovery times and increase the likelihood of recovery to dialysis independence. Lastly, a better understanding of the risks of death and dialysis dependence would help patients navigate their options regarding palliative/conservative care after having gained some experience as to what is involved in undergoing maintenance dialysis in the outpatient setting.

The optimal outpatient management of AKI-D patients remains uncertain. Currently, no evidence based-guidelines exist for the best management of AKI-D patients in order to optimize kidney recovery $[1,2]$. Many observational studies have reported on predictors of kidney recovery prior to hospital discharge [2, 5-7]. Nonetheless, some patients who require ongoing outpatient dialysis after hospital discharge will go on to recover kidney function to dialysis independence. Much less is known about risk factors that influence kidney recovery in the outpatient setting after a hospital encounter with AKI-D. Here, we present a protocol for a systematic review that will seek to assess the association between both patient- and treatment-related factors (modifiable and non-modifiable) with short- and long-term outcomes in patients who remained dialysis-dependent after a hospitalization with AKI-D.

\section{Methods \\ Eligibility criteria \\ Types of studies}

All studies that have reported on predictors of kidney recovery and clinically relevant outcomes (listed below) in AKI-D patients requiring post-hospitalization dialysis are eligible. These studies must present predictive/prognostic factors from baseline to follow-up period. In addition, studies must be full-text, peer-reviewed articles. This review will include both observational and interventional studies. Case reports or case series of less than or equal to 10 patients will be excluded. No restrictions will be placed on the study duration, study period, or date of publication. Studies must have analyzed potential predictors of kidney recovery in AKI-D patients who remain dialysisdependent after hospital discharge. Only original peerreviewed literature will be included. Studies published in all languages will be included. In addition, the following criteria must also be met:

Patient populations: Patients 18 years or older with acute kidney injury treated with dialysis of any type during a hospitalization who transition to outpatient dialysis after hospital discharge. Those with pre-existing endstage renal disease defined as estimated glomerular filtration rate $(\mathrm{eGFR})<15 \mathrm{ml}$ per minute per $1.73 \mathrm{~m}^{2}$ prior to hospitalization will be excluded.

Variables of interest or predictive factors: Any independent variable examined for potential prognostic ability will be included. We will begin with a broad study approach that spans a variety of prognostic factors which will subsequently be grouped into relevant domains. Potential domains include demographics (e.g., gender, age, ethnicity, pre-existing comorbidities), hospitalization-related factors (such as etiology of acute kidney injury, volume status at initiation of renal replacement therapy (RRT), ward versus intensive care unit admission, duration of hospitalization, urinary output at the time of hospital discharge), and treatment-related factors including modality of RRT initiated in hospital and characteristics of RRT after discharge.

\section{Outcomes}

The primary outcome of interest in this systematic review is kidney recovery to dialysis independence within 90 days after hospital discharge. This time interval is regarded as the threshold at which most patients would be considered to have reached end-stage renal disease [1]. The secondary outcomes of this study include kidney recovery to dialysis independence at any time point after hospital discharge, death at any time point after hospital discharge, progression to end-stage renal disease, and long-term RRT dependence, kidney transplantation, serum creatinine, and/or estimated glomerular filtration rate amongst those that recover to dialysis independence.

\section{Search strategy}

No prior published studies that addressed similar topics were found in PubMed, MEDLINE (via Ovid), and EMBASE (via Ovid). Similar systematic review study protocols have not been registered on PROSPERO. A health sciences librarian (LS) searched the following databases: MEDLINE and MEDLINE in Process (via OVID), Embase Classic + Embase (via OVID), Cochrane's Central Registry for Randomized Controlled Trials, CENTRAL (via OVID), and PubMed. Appendix 1 demonstrates our search strategy, which was initially developed in MEDLINE then translated into other databases. All databases will be 
searched from the date of inception to April 1, 2019. No language exclusion criteria nor other publication restrictions were incorporated into the search strategy. All references will then be entered into EndNote (version X9, Clarivate Analytics Inc., Philadelphia, PA) citation manager for processing. Supplemental search will consist of manual scanning of bibliographies in eligible studies, grey literature search of clinical trial registries such as clinicaltrials.gov, and screening for relevant titles in the first three pages of the Google Scholar search. Relevant articles will be screened at two levels: title/abstract then full-text screening, by two reviewers (CW and EC) in the systematic review software, Covidence (Veritas Health Innovation LTD).

\section{Study records}

\section{Data management}

Relevant data will be extracted from Covidence (Veritas Health Innovation LTD) and managed with Microsoft Excel. Data will be synthesized with the Comprehensive Meta-Analysis (version 2, Biostat) software.

\section{Data collection process}

Two investigators (CW and EC) will independently screen relevant study titles and abstracts per pre-specified criteria to determine their eligibilities for full-text assessment. The same investigators will subsequently examine the full texts of the selected studies according to the inclusion and exclusion criteria. Relevant studies published in English or French will be included. Disagreements will be resolved with discussion until consensus is reached. A PRISMA diagram will be generated to document the study selection process [8].

\section{Data extraction}

A data extraction form will be created and populated with the following information:

1. Patient demographics: age, gender, ethnicity.

2. Investigated independent variables/predictors:

- Patient comorbidities: baseline creatinine (if available), pre-existing chronic kidney disease, diabetes, congestive heart failure, etc.

- Hospitalization-related factors: etiology of acute kidney injury, volume status at initiation of RRT, ward versus intensive care unit admission, duration of hospitalization, urine output at the time of hospital discharge.

- Treatment-related factors: modality of RRT initiated in hospital, characteristics of outpatient RRT after discharge.

3. Outcomes: kidney recovery after hospital discharge, mortality at any time point after hospital discharge, progression to end-stage renal disease with transition to long-term RRT, eGFR at the time of kidney recovery, time from hospital discharge to kidney recovery, and kidney transplantation.

4. Study characteristics: author, publication year, number of study participants, type of study (research design), and duration of follow-up.

\section{Quality assessment}

The qualities of reporting and risk for bias of each included study will be appraised using validated tools. Prognostic studies that meet the inclusion criteria will be assessed for internal validity with the Quality In Prognostic Studies (QUIPS) tool [9]. The quality of reporting of observational studies will be assessed in accordance with Strengthening the Reporting of Observational Studies in Epidemiology (STROBE) checklist [10, 11]. The Cochrane Risk of Bias Assessment Tool for Non-Randomized Studies of Interventions (ROBINS-I) will be utilized to evaluate observational studies that assessed interventions [12]. The strength of the emerging evidence will be evaluated using the Grading of Recommendations Assessment, Development, and Evaluation (GRADE) method [13]. These assessments will be performed by two investigators (CW and $\mathrm{EC}$ ) independently, and disagreements will be resolved by consensus or involvement of a third reviewer $(\mathrm{SH})$ if required.

\section{Data synthesis}

\section{Qualitative/narrative synthesis}

A narrative synthesis will be performed to reflect directions of associations between prognostic factors and outcomes of interest. These associations will be reported as positive, negative, or none for each domain of risk factors.

\section{Quantitative synthesis}

Meta-analyses will be performed if the data is appropriate for quantitative synthesis. Minimum of two studies is required to provide data of the same prognostic factors pertaining to outcomes of interest to permit data pooling and synthesis. The strength of association between identified prognostic factors and outcomes will be quantified as odds ratios for each outcome of interest. Cochran's Q and the $I^{2}$ test will be used to examine the statistical heterogeneity between studies [13]. Publication bias will be assessed by visual examination of the funnel plot and using the Egger test [14]. Statistical analyses will be conducted using the generic inverse variance method in Review Manager software (RevMan, version 3).

\section{Protocol amendments}

Any amendments to the protocol will be outlined in an addendum made to specify and justify changes. These modifications and their justifications will also be included in the final report. 


\section{Discussion}

AKI-D during hospitalization is a frequent occurrence and is associated with poor outcomes. To date, there is a paucity of data on the epidemiology and outcomes of patients with AKI-D who remain dialysis-dependent at hospital discharge. Our proposed systematic review will synthesize what is known about the association of patient and treatment-related factors with short- and long-term outcomes in this population. We hope to shed light on modifiable prognostic factors that influence renal outcomes. The results could help inform the development of evidencebased clinical decision-making both during and after hospitalization of AKI-D patients in addition to guide patient counseling and navigate long-term care planning.

\section{Appendix 1}

Ovid Technologies, Inc. Email Service Search for: 39 and 52 and 66Results: 147

Database: Ovid MEDLINE(R) and Epub Ahead of Print, In-Process \& Other Non-Indexed Citations and Daily < 1946 to October 09, 2018 > Search Strategy:

1 exp Acute Kidney Injury/ (41734)

2 (acute adj (kidney or renal or nephr* or tubular or dialys*)).ti. (23152)

3 (acute adj2 (kidney or renal)).tw. (45381)

4 ((crescent* or progressive or anca* or acute) and (glomerul* or nephrit*)).ti. (5627)

5 ((kidney or renal) adj isch?emi*).ti. (2199)

6 *Nephritis, Interstitial/ci [Chemically Induced] (942)

7 *Hemorrhagic Fever with Renal Syndrome/ (2316)

8 (induced adj (kidney injury or renal injury)).tw. (1469)

9 Oliguria/ (1261)

10 acute nephr*.tw. (1079)

11 (pre-renal or prerenal).tw. (1032)

12 ((arf or aki) and (renal or kidney)).tw. (12162)

13 ((nephropath* and (contrast* adj (medi* or induced or agent $\left.\left.{ }^{*}\right)\right)$ ) or radiocontrast* or iodinated or crystal* or cast).mp. (463879)

14 (nephrotox* or (renal and toxi*)).ti. (9125)

15 renal tubul*.ti. (6387)

1614 or 15 (15370)

17 ci.fs. or contrast medi*.tw. or induced.mp. (2571306)

1816 and 17 (8116)

19 ((kidney or renal) adj isch?emi*).tw. (4807)

20 Kidney Tubules, Proximal/ (12488)

21 ur?emi*.ti. (14507)

22 renal inflammation.tw. (1127)

2319 or 20 or 21 or 22 (32601)

24 *Reperfusion Injury/ (19063)

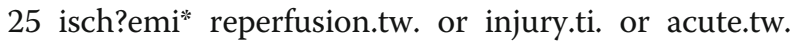
(1248297)

2624 or 25 (1250950)

2723 and 26 (7078)
28 h?emolytic ur?emi*.ti. (3812)

29 (thrombotic adj (thrombocytopeni* or microangiopathy)).tw. (6588)

3028 or 29 (9770)

31 (kidney or renal or acute).mp. (2048405)

3230 and 31 (4803)

33 (induced adj (kidney or renal)).tw. (6968)

34 (nephrotox* $^{*}$ or contrast medi* or reperfusion or perfusion).mp. (361366)

3533 and 34 (2354)

36 ((tubulointerstitial or interstitial or anti-glomerular or antiglomerular) and (glomerul* or nephrit*)).ti. (3139)

37 (acute or crescentic or atypical or progressive).mp. (1509375)

3836 and 37 (1537)

391 or 2 or 3 or 4 or 5 or 6 or 7 or 8 or 9 or 10 or 11 or 12 or 13 or 18 or 27 or 32 or 35 or 38 (547523)

40 exp Renal Replacement Therapy/ (192706)

41 ((kidney* $^{*}$ or renal) adj3 replacement adj2 therap*).tw. (11268)

42 exp Renal Dialysis/ (107143)

43 ((renal or extracorporeal) adj2 dialys\#s).tw. (2899)

44 exp Hemofiltration/ (6454)

45 h?mofiltration*.tw. (3280)

46 h?modialys\#s.tw. (57340)

47 h?modialfiltration*.tw. (19)

48 Dialysis/ (12478)

49 cavf.tw. (37)

50 sustained low-efficiency dialysis.tw. (112)

51 sled.tw. (931)

52 or/40-51 (226540)

53 Outpatients/ (13850)

54. Ambulatory Care Facilities/ (17216)

55 outpatient clinics, hospital/ (15294)

56 Surgicenters/ (1922)

57 exp General Practice/ (72426)

58 Ambulatory Care Facilities/ (17216)

59 (ambulatory adj3 care).tw. (11644)

60 (ambulatory adj2 health*).tw. (1050)

61 (surgicent* or surgi-cent*).tw. (104)

62 ((general or family) adj2 practice*).tw. (51778)

63 (outpatient* or out-patient*).tw. (163496)

64 (posthospitalization or posthospitalisation or posthospitalization or post-hospitalisation).tw. (752)

65 (postdischarg* or post discharg*).tw. (7564)

66 or/53-65 (301279)

6739 and 52 and 66 (147)

\section{Abbreviations}

AKI-D: Acute kidney injury requiring dialysis; eGFR: Estimated glomerular filtration rate; EMBASE: Excerpta Medica database; GRADE: Grading of Recommendations Assessment, Development and Evaluation;

MEDLINE: Medical Literature Analysis and Retrieval System Online; PRISMA: Preferred Reporting Items for Systematic Reviews and Meta-analyses; PROSPERO: International Prospective Register of Systematic Reviews; 
QUIPS: Quality In Prognostic Studies; ROBINS-I: Risk of Bias In Nonrandomized Studies of Interventions; RRT: Renal replacement therapy; STROBE: Strengthening the Reporting of Observational Studies in Epidemiology

\section{Acknowledgements}

The authors acknowledge the institutional support of the Kidney Research Centre, Ottawa Hospital Research Institute, University of Ottawa, Ottawa, Ontario, Canada.

\section{Authors' contributions}

CW and EC generated the systematic review protocol and wrote the manuscript. LS provided assistance by executing the search strategies. All authors revised the manuscript and read and approved the final manuscript.

\section{Funding}

Not applicable.

\section{Availability of data and materials}

The datasets used and/or analyzed during the current study are available from the corresponding author on reasonable request.

\section{Ethics approval and consent to participate}

Not applicable.

\section{Consent for publication}

Not applicable.

\section{Competing interests}

The authors declare that they have no competing interests.

\section{Author details}

'Department of Medicine, University of Ottawa, CPC 162737 Parkdale Avenue, Ottawa, Ontario K1Y 1 J8, Canada. ${ }^{2}$ Division of Nephrology, Department of Medicine, The Ottawa Hospital and University of Ottawa, The Ottawa Hospital - Riverside Campus, 1967 Riverside Drive, Ottawa, Ontario K1H 7 W9, Canada. ${ }^{3}$ Health Sciences Library, University of Ottawa, 451 Smyth Road, Ottawa, Ontario K1H 8 M5, Canada. ${ }^{4}$ Division of Nephrology, Ottawa Hospital Research Institute, The Ottawa Hospital, 1967 Riverside Drive, Ottawa, Ontario K1H 7 W9, Canada.

Received: 2 April 2019 Accepted: 13 August 2019

Published online: 22 August 2019

\section{References}

1. Cerda J, Liu KD, Cruz DN, Jaber BL, Koyner JL, Heung M, et al. Promoting kidney function recovery in patients with AKI requiring RRT. Clin J Am Soc Nephrol. 2015;10(10):1859-67.

2. Pajewski R, Gipson P, Heung M. Predictors of post-hospitalization recovery of renal function among patients with acute kidney injury requiring dialysis. Hemodial Int. 2018;22:66-73.

3. Pavkov ME, Harding JL, Burrows NR. Trends in hospitalizations for acute kidney injury: United States, 2000-2014. MMWR Morb Mortal Wkly Rep. 2018; 67(10):289-93.

4. Wald $\mathrm{R}$, et al. Changing incidence and outcomes following dialysis-requiring acute kidney injury among critically ill adults: a population-based cohort study. Am J Kidney Dis. 2015;65(6):870.

5. Gautam SC, Brooks CH, Balogun RA, Xin W, Ma JZ, Abdel-Rahman EM. Predictors and outcomes of post-hospitalization dialysis dependent acute kidney injury. Nephron. 2015;131:185-90.

6. Hickson LJ, Chaudhary S, Williams AW, Dillon JJ, Norby SM, Gregoire JR, et al. Predictors of outpatient kidney function recovery among patients who initiate hemodialysis in the hospital. Am J Kidney Dis. 2015;65(4):592-602.

7. Rathore AS, Chopra T, Ma JZ, Xin W, Abdel-Rahman EM. Long-term outcomes and associated risk factors of post-hospitalization dialysis dependent acute kidney injury patients. Nephron. 2017;137(2):105-12.

8. Shamseer L, Moher D, Clarke M, Ghersi D, Liberati A, Petticrew M, et al. Preferred reporting items for systematic review and meta-analysis protocols (PRISMA-P) 2015: elaboration and explanation. BMJ. 2015;350:g7647.

9. Hayden JA, van der Windt DA, Cartwright JL, Cote P, Bombarider C. Assessing bias in studies of prognostic factors. Ann Intern Med. 2013;158:280-6.
10. Sterne JAC, Higgins JPT, Reeves BC on behalf of the development group for ACROBAT-NRSI. A Cochrane Risk Of Bias Assessment Tool: for Non-Randomized Studies of Interventions (ACROBAT-NRSI), Version 1.0.0, 24 September 2014. Available from http://www.riskofbias.info [accessed 15 Aug 2018].

11. von Elm E, Altman DG, Egger M, Pocock SJ, Gøtzsche PC, Vandenbroucke JP, Initiative STROBE. The Strengthening the Reporting of Observational Studies in Epidemiology (STROBE)statement: guidelines for reporting observational studies. J Clin Epidemiol. 2008 Apr;61(4):344-9.

12. Sterne JA, Hernan MA, Reeves BC, Savovic J, Berkman ND, Viswanathan M, et al. ROBINS-l: a tool for assessing risk of bias in non-randomized studies of interventions. BMJ. 2016:355:i4919.

13. Higgins JPT, Green S (editors). Cochrane Handbook for Systematic Reviews of Interventions Version 5.1.0 [updated March 2011]. The Cochrane Collaboration, 2011. Available from www.handbook.cochrane.org.

14. Egger M, Davey Smith G, Schneider M, Minder C. Bias in meta-analysis detected by a simple, graphical test. BMJ. 1997 Sep 13;315(7109):629-34.

\section{Publisher's Note}

Springer Nature remains neutral with regard to jurisdictional claims in published maps and institutional affiliations.

\section{Ready to submit your research? Choose BMC and benefit from:}

- fast, convenient online submission

- thorough peer review by experienced researchers in your field

- rapid publication on acceptance

- support for research data, including large and complex data types

- gold Open Access which fosters wider collaboration and increased citations

- maximum visibility for your research: over $100 \mathrm{M}$ website views per year

At BMC, research is always in progress.

Learn more biomedcentral.com/submissions 